\title{
Association between sleep insufficiency and dyslipidemia: a cross- sectional study among Greek adults in the primary care setting
}

\author{
Dimitrios Tsiptsios ${ }^{1 *}$ \\ Eleni Leontidou ${ }^{2}$ \\ Petros N. Fountoulakis ${ }^{3}$ \\ Andreas Ouranidis ${ }^{4}$ \\ Anestis Matziridis ${ }^{2}$ \\ Apostolos Manolis ${ }^{2}$ \\ Andreas S. Triantafyllis ${ }^{3}$ \\ Konstantinos Tsamakis ${ }^{5}$ \\ Aspasia Serdari ${ }^{6}$ \\ Aikaterini Terzoudi ${ }^{7}$ \\ Elena Dragioti ${ }^{8}$ \\ Paschalis Steiropoulos ${ }^{9}$ \\ Gregory Tripsianis ${ }^{2}$
}

${ }^{1}$ South Tyneside \& Sunderland NHS Foundation Trust, Department of Clinical Neurophysiology - Sunderland Tyne \& Wear - United Kingdom.

${ }^{2}$ Democritus University of Thrace, Laboratory of Medical Statistics Alexandroupolis - Thrace - Greece. ${ }^{3}$ Askepeion Hospital, Department of Cardiology - Athens - Attiki - Greece. ${ }^{4}$ Aristotle University of Thessaloniki, Department of Pharmaceutics -

Thessaloniki - Central Macedonia Greece.

${ }^{5}$ King's College, Institute of Psychiatry, Psychology and Neuroscience - London - London - United Kingdom.

${ }^{6}$ Democritus University of Thrace,

Department of Child and Adolescent Psychiatry - Alexandroupolis - Thrace - Greece.

${ }^{7}$ Democritus University of

Thrace, Neurology Department -

Alexandroupolis - Thrace - Greece.

${ }^{8}$ Linköping University, Department of Health, Medicine and Caring Sciences Linköping - Linköping - Sweden.

${ }^{9}$ Democritus University of Thrace,

Department of Pneumonology -

Alexandroupolis - Thrace - Greece.

*Corresponding author:

Dimitrios Tsiptsios

E-mail: tsiptsios.dimitrios@yahoo.gr /

tsiptsios.dimitrios@gmail.com

Received: December 29, 2020;

Accepted: March 8, 2021.

\begin{abstract}
Objective: To investigate the potential association between sleep insufficiency and dyslipidemia (DL) in the primary care setting using self-reported questionnaires. Material and Methods: 957 adults aged between 19 and 86 years old from the rural area of Thrace, Greece were enrolled in this cross-sectional study. Multistage stratified cluster sampling was used and the subjects were classified into three groups according to sleep duration [short $(<6 \mathrm{~h})$, normal $(6-8 \mathrm{~h})$, and long $(>8 \mathrm{~h})$ sleep duration]. DL was defined by a positive response to the question "Have you ever been told by a doctor or health professional that your blood cholesterol or triglyceride levels were high?”, or if they were currently taking antilipidemic agents. Sleep quality, utilizing Epworth sleepiness scale, Athens insomnia scale, Pittsburgh sleep quality index and Berlin questionnaire, was also examined. Results: DL prevalence was significantly associated with short sleep duration $(\mathrm{aOR}=2.18, \mathrm{p}<0.001)$ and insomnia $(\mathrm{aOR}=1.43, \mathrm{p}=0.050)$, while its relation with poor sleep quality $(\mathrm{aOR}=1.31, \mathrm{p}=0.094)$ and risk for obstructive sleep apnea $(\mathrm{aOR}=1.32, \mathrm{p}=0.097)$ were of marginal statistical significance. Concerning insomnia subtypes, DL was significantly associated with difficulties maintaining sleep $(\mathrm{aOR}=2.99, \mathrm{p}<0.001)$ and early morning awakenings $(\mathrm{aOR}=1.38, \mathrm{p}=0.050)$, but not difficulties initiating sleep $(\mathrm{aOR}=1.18, \mathrm{p}=0.328)$. Conclusion: This study reveals an association between sleep pathology and DL. Thus, early pharmacological and cognitive or behavioral interventions that improve sleep are deemed necessary in order to decrease DL burden.
\end{abstract}

Keywords: Cross-Sectional Study; Sleep Duration; Dyslipidemia; Sleep Quality; Insomnia. 


\section{INTRODUCTION}

Cardiovascular disease (CVD) comprises a major public health issue, as it constitutes the leading cause of noncommunicable disease mortality worldwide ${ }^{1}$. CVD pathogenesis is complex including non-modifiable risk factors, such as age, gender, race and ethnicity, and modifiable risk factors, such as smoking, lack of physical activity, diabetes mellitus, hypertension, and visceral obesity ${ }^{2}$. Dyslipidemia (DL) defined as high levels of total cholesterol (TC), low-density lipoprotein cholesterol (LDL-C), triglycerides (TG), and low levels of high-density lipoprotein cholesterol (HDL-C), is another wellestablished reversible risk factor for CVD, thus encompassing an important issue in the field of health promotion and CVD prevention ${ }^{3,4}$.

Serum lipid and lipoprotein levels are influenced by lifestyle parameters, such as smoking, alcohol consumption, and exercise $^{5,6}$. Due to public health concerns about the declining quality and quantity of sleep in modern society, research into the effect of sleep on health has blossomed. Although in recent years, both short and long sleep duration have been associated with chronic diseases such as hypertension ${ }^{7}$, diabetes mellitus ${ }^{8}$, and $\mathrm{CVD}^{9}$, results on the potential relationship between sleep duration and DL have been contradictory so far, as U-shaped associations ${ }^{10,11}$ or relationships between DL and only short ${ }^{12}$ or long sleep duration ${ }^{13}$ have been presented. Apart from that, few studies have investigated the effect of sleep quality on DL reaching antithetical conclusions, as well ${ }^{14-16}$. Furthermore, there exists no consensus on the probable gender-specific association between sleep pathology and DL ${ }^{17,18}$. Finally, few European studies have investigated the potential association between sleep pathology and $\mathrm{DL}^{12,19}$.

Our research group utilizing self-reported questionnaires has recently exhibited that sleep pathology is associated with increased prevalence of anxiety ${ }^{20}$, depression ${ }^{21}$, diabetes mellitus $^{22}$, and hypertension ${ }^{23}$. In this paper, we aimed to investigate possible correlations between sleep quantity/ quality and DL considering several socio-demographic characteristics, lifestyle habits and health related characteristics of the participants and focusing on potential gender-specific associations.

\section{MATERIAL AND METHODS}

\section{Study sample, research design and covariates}

The study population in this cross-sectional study consisted of 957 participants, $439(45.9 \%)$ males and 518 (54.1\%) females, with a mean age of $49.62 \pm 14.79$ years (range, 19-86 years; median age, 50 years). The research design of this study is reported in Serdari et al. $(2020)^{20}$. The questionnaires used in order to collect standard socio-demographic characteristics, lifestyle, and dietary habits and health related characteristics of the participants are reported in Matziridis et al. (2020) ${ }^{22}$. Chronic disease morbidity was defined as the self-reported preexisting (for $\geq 3$ months over the past year) health problems, such as: hypertension, diabetes mellitus, cancer, cardiovascular, rheumatic, gastrointestinal, pulmonary, or neurologic disease.

\section{ETHICS}

All procedures performed in the study were in accordance with the ethical standards of the Democritus University Ethics Committee, which approved its conduct, and with the standards of the Helsinki declaration (1964) and its later amendments. Informed consent was obtained from all participants of the study.

\section{Estimation of sleep duration and sleep efficiency}

Participants answered the following questions: "At what time do you normally go to bed?", "At what time do you normally get up?", and "On average, how many hours do you sleep every night?" for an average weekday and weekend day over the previous month. Time in bed, sleep duration, and sleep efficiency calculation formulae on weekdays, weekend days and weighted mean measures utilized in this study are reported in Matziridis et al. (2020) $)^{22}$. According to calculated sleep duration, participants were then classified into: short sleepers ( $<6$ hours), normal sleepers (6-8 hours), and long sleepers $(>8 \text { hours })^{24,25}$.

\section{Evaluation of sleep quality}

Sleep quality was assessed with the Greek versions of Epworth sleepiness scale (ESS) ${ }^{26}$, Athens insomnia scale $(\mathrm{AIS})^{27}$, Pittsburgh sleep quality index (PSQI) ${ }^{28}$, and Berlin Questionnaire $(\mathrm{BQ})^{29}$ that evaluate excessive daytime sleepiness, insomnia, sleep quality, and risk of obstructive sleep apnea (OSA), respectively. With regards to insomnia characteristics, participants were asked whether they experienced difficulties initiating or maintaining sleep or early morning awakenings.

\section{Definition of DL}

DL was defined by a positive response to the question "Have you ever been told by a doctor or health professional that your blood cholesterol or triglyceride levels were high?" or if they were currently taking antilipidemic agents ${ }^{30,31}$.

\section{Statistical analysis}

Statistical analysis of the data was performed using IBM Statistical Package for the Social Sciences (SPSS), version 19.0 (IBM Corp., Armonk, NY, U.S.A.). The normality of quantitative variables was tested with Kolmogorov-Smirnov test. Quantitative variables were expressed as mean \pm standard deviation $(\mathrm{SD})$ and qualitative variables were expressed as absolute and relative (\%) frequencies. In particular, mean estimated time of sleep characteristics (i.e., bedtime, rise time, time in bed, and sleep duration) were expressed as HH:MM. We conducted the following analyses: (i) in the univariate analysis, the association of DL with subjects' characteristics, sleep characteristics, and sleep disorders were assessed using the chi-square test and Student's t-test; (ii) multivariate stepwise logistic regression analysis was used to explore the independent risk factors for DL, controlling for all subjects' characteristics; (iii) for the evaluation of the 
effect of sleep duration and sleep disorders on the prevalence of DL, two different logistic regression models were constructed: model 1 (crude, unadjusted) and model 2 (adjusted for subjects' socio-demographic characteristics: gender, age, marital status, cultural status, place of residence, education level, working status, financial status; lifestyle habits: smoking status, alcohol consumption, daily coffee consumption, caffeine consumption in the evening, adherence to the Mediterranean diet, time watching TV or using a computer before bedtime, physical activity, nap during the day; and health related characteristics: subjective general health status, BMI, chronic disease morbidity, anxiety, depression, and use of sleep medication). Odds ratios (OR) with their 95\% confidence intervals (CI) were estimated as the measure of the above associations.
Receiver operating characteristic (ROC) analysis was used to provide the ability of sleep duration to classify subjects with DL. The area under the ROC curve (AUC), sensitivity, and specificity were estimated. The optimal cutoff value of the sleep duration that differentiates DL from non-DL individuals was derived according to Youden index. All tests were two tailed and statistical significance was considered for $p$-values $\leq 0.05$.

\section{RESULTS}

\section{Subjects' characteristics}

Participants' socio-demographic, lifestyle and health related characteristics are summarized in Tables 1 and 2. The

Table 1. Prevalence of dyslipidemia in relation to subjects' demographic characteristics.

\begin{tabular}{|c|c|c|c|c|}
\hline & \multicolumn{4}{|c|}{ Dyslipidemia } \\
\hline & Total sample & Frequency & Proportion (\%) & $p$-value \\
\hline Gender & & & & 0.310 \\
\hline Females & $518(54.1)$ & 116 & 31.1 & \\
\hline Males & $439(45.9)$ & 150 & 34.2 & \\
\hline Age (years) & & & & $<0.001$ \\
\hline$\leq 30$ & $132(13.8)$ & 9 & 6.8 & \\
\hline $30-40$ & $141(14.7)$ & 24 & 17.0 & \\
\hline $41-50$ & $232(24.2)$ & 51 & 22.0 & \\
\hline $51-60$ & $212(22.2)$ & 71 & 33.5 & \\
\hline $61-70$ & $145(15.2)$ & 81 & 55.9 & \\
\hline$>70$ & $95(9.9)$ & 75 & 78.9 & \\
\hline Marital status & & & & $<0.001$ \\
\hline Married & $645(67.4)$ & 220 & 34.1 & \\
\hline Single & $196(20.5)$ & 23 & 11.7 & \\
\hline Divorced & $36(3.8)$ & 8 & 22.2 & \\
\hline Widowed & $80(8.4)$ & 60 & 75.0 & \\
\hline Cultural status & & & & 0.020 \\
\hline Greek Christians & $632(66.1)$ & 189 & 29.9 & \\
\hline Greek Muslims & $273(28.5)$ & 107 & 39.2 & \\
\hline Expatriated Greeks & $52(5.4)$ & 15 & 28.8 & \\
\hline Place of residence & & & & $<0.001$ \\
\hline Urban & $416(43.5)$ & 84 & 20.2 & \\
\hline Rural & $541(56.5)$ & 227 & 42.0 & \\
\hline Education level & & & & $<0.001$ \\
\hline Low & $313(32.7)$ & 158 & 50.5 & \\
\hline Medium & $340(35.5)$ & 98 & 28.8 & \\
\hline High & $304(31.8)$ & 55 & 18.1 & \\
\hline Working Status & & & & 0.927 \\
\hline Employed & $872(91.1)$ & 283 & 32.5 & \\
\hline Unemployed & $85(8.9)$ & 28 & 32.9 & \\
\hline Financial status $(n=812)$ & & & & $<0.001$ \\
\hline Low & $476(49.7)$ & 183 & 38.4 & \\
\hline Medium & $200(20.9)$ & 68 & 34.0 & \\
\hline High & $136(14.2)$ & 20 & 14.7 & \\
\hline
\end{tabular}


Table 2. Prevalence of dyslipidemia in relation to subjects' lifestyle habits and health related characteristics.

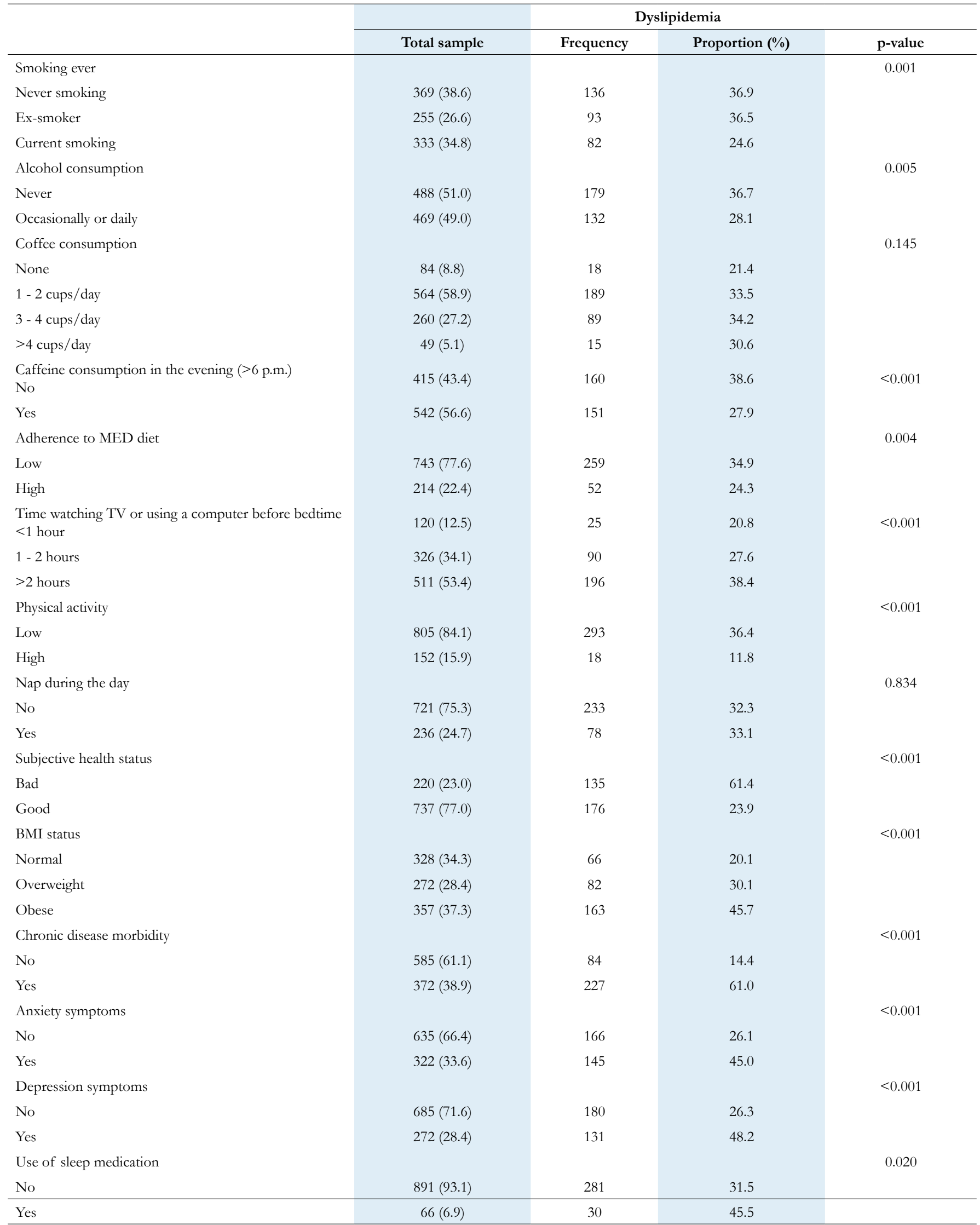


average self-reported sleep duration was 6 hrs and $19 \mathrm{~min}$ on the weekdays and $6 \mathrm{hrs}$ and $45 \mathrm{~min}$ on the weekends; $31.7 \%$ and $22.9 \%$ of the sample reported sleep duration less than $6 \mathrm{hrs}$, while $7.9 \%$ and $14.2 \%$ of the sample reported sleep duration more than $8 \mathrm{hrs}$ per night on the weekdays and on the weekends, respectively. Sixty-six participants $(6.9 \%)$ were on sleep related medications.

A total of 311 participants (32.5\%) were classified with DL. The prevalence of DLin relation to participants' socio-demographic, lifestyle and health related characteristics is summarized in Tables 1 and 2. Significant determinants of DL obtained by multivariate logistic regression models are presented in Table 3 .

Table 3. Significant determinants of dyslipidemia obtained by multivariate logistic regression models.

\begin{tabular}{lccc}
\hline Characteristics & aOR & $\mathbf{9 5 \%} \mathbf{C I}$ & $p$-value \\
\hline Age (10-year increase) & 1.84 & $1.59-2.13$ & $<0.001$ \\
Widowed & 2.74 & $1.44-5.20$ & 0.002 \\
Low education level & 2.69 & $1.47-4.91$ & 0.001 \\
Low or medium financial status & 1.91 & $1.10-3.31$ & 0.022 \\
Never or ex-smoking & 1.67 & $1.12-2.49$ & 0.011 \\
No alcohol consumption & 1.50 & $1.04-2.18$ & 0.031 \\
Low adherence to & 1.67 & $1.09-2.57$ & 0.019 \\
Mediterranean diet & 2.58 & $1.79-3.70$ & $<0.001$ \\
Obesity & 2.71 & $1.93-3.81$ & $<0.001$ \\
\hline Anxiety symptoms & & & \\
\hline
\end{tabular}

Notes: $\mathrm{aOR}=$ Adjusted odds ratio; $\mathrm{CI}=$ Confidence interval; all subjects' sociodemographic, lifestyle habits and health related characteristics were included in the model; All variables (with the exception of age) were binary (no, yes); category "no" forms the reference group.

\section{DL and sleep habits}

The association of DL with subjects' sleep characteristics is shown in Table 4. The average weekly time in bed and sleep duration were calculated and compared between the two groups. It was noted that, although subjects with DL spent $11 \mathrm{~min}$ longer time in bed $(p=0.007)$, they reported a $22 \mathrm{~min}$ shorter sleep duration $(p<0.001)$ and lower sleep efficiency $(p<0.001)$ compared to those without DL. All the above relations between DL and sleep characteristics remained unchanged among females and males. In particular, females with DL used to sleep 21 min less than females without DL $(p=0.005)$ and males with DL used to sleep $22 \mathrm{~min}$ less than males without DL ( $p=0.003)$. Among subjects with DL, all three sleep characteristics were similar between males and females (time in bed: $p=0.085$; sleep duration: $p=0.303$; sleep efficiency: $p=0.964$ ).

In the sequence, according to the self-reported sleep duration, participants were categorized into three groups: short $(<6 \mathrm{~h})$, normal $(6-8 \mathrm{~h})$, and long $(>8 \mathrm{~h})$ sleep duration. The association between the development of DL and sleep duration, which was considered as a categorical variable (Table 5) revealed that DL was significantly more frequent $(p<0.001)$ in subjects with short $(53.1 \%)$ compared to those with normal $(25.8 \%)$ and long $(30.9 \%)$ sleep duration. The association of DL with sleep duration status had the same pattern in both genders $(p=0.002$ for females; $p<0.001$ for males) (Table 5). In particular, logistic regression analysis revealed that in subjects with short sleep duration there were more than 3-times higher odds for DL compared to subjects with normal sleep duration $(\mathrm{OR}=3.26$, $p<0.001)$. A 2.65-fold $(p<0.001)$ and a 3.92-fold $(p<0.001)$ increase in odds of DL was associated with short sleep duration in women and men, respectively.

\section{Independent effect of DL on sleep habits}

Two separate multivariate logistic regression models, controlling for the effect of all subjects' socio-demographic, lifestyle, and health related characteristics, were constructed in order to assess the independent effect of sleep duration on the prevalence of DL. When sleep duration was entered in the model as a continuous variable, it remained a statistically significant independent determinant of DL ( $\phi=0.049)$; in particular, shorter sleep duration by one hour was associated with a $14 \%$-increase in the odds for $\mathrm{DL}(\mathrm{aOR}=1.14,95 \% \mathrm{CI}=1.00-1.30)$.

When sleep duration was entered in the multivariate logistic regression model as a categorical variable, the inverse relationship between DL and sleep duration persisted even after the adjustment for all subjects' characteristics. In particular, the odds of DL were more than 2 times higher in subjects sleeping less than 6 hours $(\mathrm{aOR}=2.18, p<0.001)$ relative to those with normal sleep duration; the respective odds for DL were similar in the two genders $(\mathrm{aOR}=2.01, p=0.023$ in females; $\mathrm{aOR}=2.84$, $p<0.001$ in males). Sleeping longer than 8 hours showed no significant effect on the development of DL (Table 5).

Moreover, the area under the ROC curve (AUC) showed that sleep duration has a significant ability to discriminate subjects with DL (AUC $=0.633,95 \% \mathrm{CI}=0.592-0.675, p<0.001$ ). The optimal cutoff point of sleep duration of 5:33 hours, which was determined to classify subjects with DL, yielded high sensitivity of $50 \%$ and specificity of $85 \%$. Sleep duration showed significant discrimination ability in both genders, although its performance was superior among males (females: AUC $=0.578,95 \%$ CI $=0.520-0.637, p=0.005$, cutoff $\leq 5: 33$ hours, sensitivity $=39 \%$, and specificity $=85 \%$; males: $\mathrm{AUC}=0.688,95 \%$ $\mathrm{CI}=0.630-0.746, p=0.030$, cutoff $\leq 5: 38$ hours, sensitivity $=60 \%$, and specificity $=86 \%$ ).

\section{DL and sleep disorders}

According to the Greek versions of ESS, AIS, PSQI and BQ the prevalence of daytime sleepiness was $8.7 \%$ (83 subjects), insomnia 18.0\% (172 subjects), poor sleep quality 38.5\% (368 subjects), and high risk of obstructive sleep apnea 36.4\% (348 subjects). The internal consistency of all four questionnaires was very high (Cronbach $\alpha$ coefficient was ranged from 0.74 to 0.88$)$. The presence of DL in relation to sleep disorders is shown in Table 6.

Univariate statistical analysis showed that DL was more frequent in subjects with insomnia ( $40.7 \%$ vs. $30.7 \%, p=0.011)$, poor sleep quality $(43.2 \%$ vs. $25.8 \%, p<0.001)$ and high risk for OSA $(39.1 \%$ vs. $28.7 \%, p=0.001)$; no association with excessive daytime sleepiness was found $(p=0.466)$.

In multivariate logistic regression analysis controlling for all subjects' characteristics, the odds of DL remained significantly 
Table 4. Association of dyslipidemia with sleep characteristics.

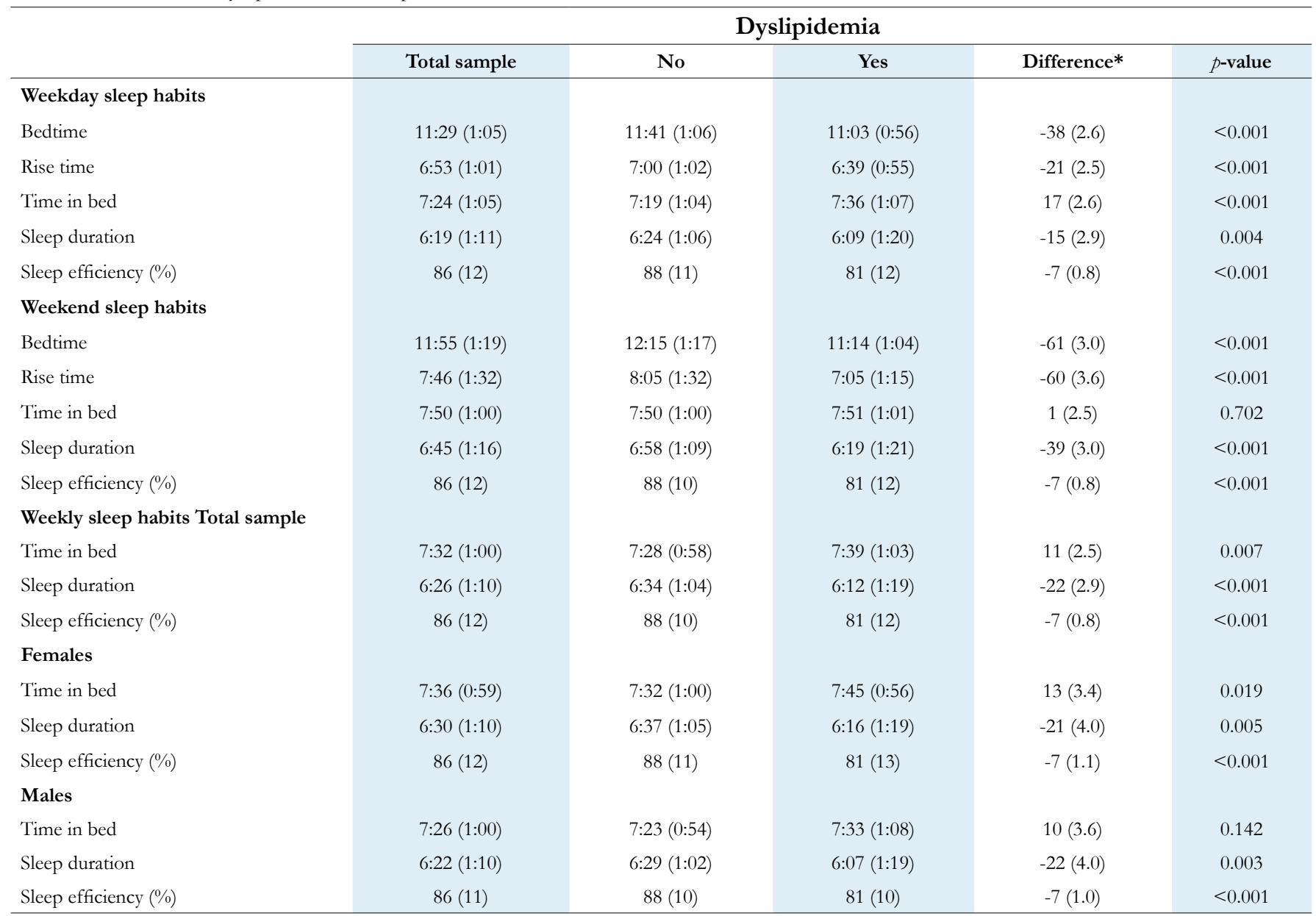

Notes: *mean difference (S.E.) between subjects with and without dyslipidemia is expressed as minutes (bedtime, rise time, time in bed, and sleep duration) and percentages (sleep efficiency).

Table 5. Association of sleep duration with dyslipidemia (DL) using logistic regression models.

\begin{tabular}{|c|c|c|c|c|c|c|}
\hline & & & Model 1 & & Model 2 & \\
\hline & DL, n (\%) & p-value & $\operatorname{cOR}(95 \% \mathrm{CI})$ & p-value & aOR $(95 \% \mathrm{CI})$ & p-value \\
\hline \multicolumn{7}{|l|}{ Total } \\
\hline Sleep duration & & $<0.001$ & & & & \\
\hline Short & $111(53.1)$ & & $3.26(2.35-4.51)$ & $<0.001$ & $2.18(1.50-3.19)$ & $<0.001$ \\
\hline Normal & $158(25.8)$ & & Ref. & & Ref. & \\
\hline Long & $42(30.9)$ & & $1.28(0.86-1.93)$ & 0.228 & $1.02(0.64-1.64)$ & 0.932 \\
\hline \multicolumn{7}{|l|}{ Females } \\
\hline Sleep duration & & 0.002 & & & & \\
\hline Short & $45(48.9)$ & & $2.65(1.65-4.25)$ & $<0.001$ & $2.01(1.10-3.68)$ & 0.023 \\
\hline Normal & 94 (26.6) & & Ref. & & Ref. & \\
\hline Long & $22(30.6)$ & & $1.22(0.70-2.12)$ & 0.487 & $1.34(0.70-2.59)$ & 0.384 \\
\hline \multicolumn{7}{|l|}{ Males } \\
\hline Sleep duration & & $<0.001$ & & & & \\
\hline Short & $66(56.4)$ & & $3.92(2.47-6.23)$ & $<0.001$ & $2.84(1.69-4.75)$ & $<0.001$ \\
\hline Normal & $64(24.8)$ & & Ref. & & Ref. & \\
\hline Long & $20(31.1)$ & & $1.38(0.76-2.51)$ & 0.295 & $0.91(0.47-1.77)$ & 0.787 \\
\hline
\end{tabular}

Notes: $\mathrm{cOR}=$ Crude odds ratio; $\mathrm{aOR}=$ Adjusted odds ratio; $\mathrm{CI}=$ Confidence interval; Model 1 = Crude, unadjusted; Model $2=$ Adjusted for socio-demographic characteristics, lifestyle habits (smoking status, alcohol consumption, daily coffee consumption, caffeine consumption in the evening, adherence to the Mediterranean diet, time watching TV or using a computer before bedtime, physical activity, nap during the day) and health related characteristics (subjective general health status, BMI, chronic disease morbidity, anxiety, depression, and use of sleep medication). 
Table 6. The association of sleep questionnaires and sleep difficulties with dyslipidemia (DL) using logistic regression models.

\begin{tabular}{|c|c|c|c|c|c|c|}
\hline & & & Model 1 & & Model 2 & \\
\hline & DL & $p$-value & $\operatorname{cOR}(95 \% \mathrm{CI})$ & $p$-value & aOR $(95 \% \mathrm{CI})$ & $p$-value \\
\hline \multicolumn{7}{|l|}{ Sleep questionnaires } \\
\hline ESS & & 0.466 & & & & \\
\hline Normal day sleepiness & $287(32.8)$ & & Ref. & & Ref. & \\
\hline Excessive day sleepiness & $24(28.9)$ & & $0.83(0.51-1.37)$ & 0.466 & $0.72(0.44-1.20)$ & 0.212 \\
\hline AIS & & 0.011 & & & & \\
\hline Non-insomniac & $241(30.7)$ & & Ref. & & Ref. & \\
\hline Insomniac & $70(40.7)$ & & $1.59(1.10-2.8)$ & 0.011 & $1.43(1.00-2.03)$ & 0.050 \\
\hline PSQI & & $<0.001$ & & & & \\
\hline Good quality & $152(25.8)$ & & Ref. & & Ref. & \\
\hline Poor quality & $159(43.2)$ & & $2.19(1.66-2.89)$ & $<0.001$ & $1.31(0.96-1.81)$ & 0.094 \\
\hline BQ & & 0.001 & & & & \\
\hline Low risk & $175(28.7)$ & & Ref. & & Ref. & \\
\hline High risk & $136(39.1)$ & & $1.59(1.21-2.10)$ & 0.001 & $1.32(0.95-1.83)$ & 0.097 \\
\hline \multicolumn{7}{|l|}{ Sleep difficulties } \\
\hline Delay in falling asleep & & 0.064 & & & & \\
\hline Less than once a week & $183(30.3)$ & & Ref. & & Ref. & \\
\hline At least once a week & $128(36.2)$ & & $1.30(0.99-1.72)$ & 0.064 & $1.18(0.85-1.63)$ & 0.328 \\
\hline Inability to maintain asleep & & $<0.001$ & & & & \\
\hline Less than once a week & $51(14.1)$ & & Ref. & & Ref. & \\
\hline At least once a week & $260(43.7)$ & & $4.73(3.38-6.63)$ & $<0.001$ & $2.99(2.05-4.36)$ & $<0.001$ \\
\hline Early morning awakenings & & $<0.001$ & & & & \\
\hline Less than once a week & $156(28.0)$ & & Ref. & & Ref. & \\
\hline At least once a week & $155(38.8)$ & & $1.63(1.24-2.14)$ & $<0.001$ & $1.38(1.00-1.89)$ & 0.050 \\
\hline
\end{tabular}

Notes: ESS = Epworth sleepiness scale; AIS = Athens insomnia scale; PSQI = Pittsburgh sleep quality index; BQ = Berlin questionnaire; $\mathrm{cOR}=\mathrm{Crude}$ odds ratio; $\mathrm{aOR}=\mathrm{Adjusted}$ odds ratio; $\mathrm{CI}=$ Confidence interval; Model 1 = Crude, unadjusted; Model 2 = Adjusted for socio-demographic characteristics, lifestyle habits, and health related characteristics.

associated only with insomnia $(\mathrm{aOR}=1.43, p=0.050)$, while its relation with poor sleep quality ( $\mathrm{aOR}=1.31, p=0.094$ ) and the risk for OSA $(\mathrm{aOR}=1.32, p=0.097)$ were of marginal statistical significance (Table 6). However, in stratified analysis according to gender, higher odds of DL were significantly associated with insomnia $(\mathrm{aOR}=1.75,95 \%$ $\mathrm{CI}=1.05-2.94, p=0.030$ ), poor sleep quality $(\mathrm{aOR}=2.02,95 \% \mathrm{CI}=1.23$ $3.33, p=0.006)$ and the risk for OSA $(\mathrm{aOR}=1.62,95 \% \mathrm{CI}=0.97-2.72$, $p=0.067)$ among women but not among men $(\mathrm{aOR}=1.18, p=0.543$ for insomnia; $\mathrm{aOR}=1.39, p=0.112$ for poor sleep quality; $\mathrm{aOR}=0.99$, $p=0.960$ for the risk for OSA).

Regarding to the basic difficulties of sleep patterns (Table 6), significant increased odds of DL were found among subjects who reported difficulties maintaining sleep $(\mathrm{aOR}=2.99, p<0.001)$ and early morning awakenings ( $\mathrm{aOR}=1.38, p=0.050)$, but not difficulties initiating sleep $(\mathrm{aOR}=1.18, p=0.328)$. Again, higher odds for $\mathrm{DL}$ were associated with difficulties maintaining sleep $(\mathrm{aOR}=6.84,95 \%$ $\mathrm{CI}=3.86-12.11, p<0.001$ ), early morning awakenings $(\mathrm{aOR}=1.70$, $95 \% \quad \mathrm{CI}=1.08-2.68, \quad p=0.022)$ and difficulties initiating sleep $(\mathrm{aOR}=1.54,95 \% \mathrm{CI}=0.94-2.52, p=0.089)$ among females, although the latter was of marginal significance, but not among males $(\mathrm{aOR}=0.79,95 \% \mathrm{CI}=0.50-1.27, p=0.331$ for difficulties initiating sleep; $\mathrm{aOR}=1.37,95 \% \mathrm{CI}=0.77-2.44, p=0.291$ for difficulties maintaining sleep and $\mathrm{aOR}=1.10,95 \% \mathrm{CI}=0.67-1.77, p=0.715$ for early morning awakenings).

\section{DISCUSSION}

A population based cross-sectional study utilizing selfreported questionnaires was conducted in the primary care setting in northeastern Greece and presented significant associations between short sleep duration and DL among adults irrespective of gender. Moreover, it was revealed that DL prevalence was significantly associated with insomnia, particularly with difficulties maintaining sleep and early morning awakenings.

The high-evidenced prevalence of DL $(32.5 \%$ of the study population sampled) is consistent with the results of Merekoulias et al. $(2014)^{32}$ who reported a prevalence of $35.7 \%$ of DL among individuals over 70 years old with poor sleep quality in Western Greece. Furthermore, DL was more prominent among Greek Muslims affecting 39.2\% of our population, compared to Expatriated Greeks and Greek Christians with proportions of $28.8 \%$ and $29.9 \%$, respectively. Several studies have also underlined the growing prevalence of DL in minority groups ${ }^{33,34}$. Moreover, rural citizens presented DL twice more frequently when compared to inhabitants of urban areas. This finding probably derives from educational and socioeconomic disparities; hence, people with low educational status from urban areas seem to be more prone to the detrimental effect of DL. Indeed, low financial and educational status in a Korean population favored the development of 
$\mathrm{DL}^{35,36}$. Quite interestingly, the marital status affected the distribution of DL in our sample, with the widowed patients holding the lion's share, as also proved in the research of Nikparvar et al. (2020) ${ }^{37}$. Additionally, our results demonstrated that factors such as ex-smoking, obesity, sedentary life, and unhealthy diets may contribute to higher DL rates of developing populations, along with previous studies ${ }^{38,39}$. Finally, in keeping with our results emerging evidence from the study of Rekleiti et al. (2012) ${ }^{40}$ demonstrated the independence of DL as a prognostic factor for anxiety.

With regards to sleep duration, our study revealed that short sleepers $(<6 \mathrm{~h})$ exhibit more than two-fold higher odds for DL compared to normal sleepers (6-8h) $(\mathrm{aOR}=2.18, p<0.001)$. Furthermore, reduced sleep duration by one hour was associated with a $14 \%$ increase in the risk for DL $(\mathrm{aOR}=1.14,95 \% \mathrm{CI}=1.00$ 1.30). Apart from that, sleep duration exhibited significant ability to discriminate subjects with DL (AUC $=0.633,95 \% \mathrm{CI}=0.592$ $0.675, p<0.001)$ determining the optimal cutoff point of $5: 33$ hours sleep duration. In contrast, sleeping longer than 8 hours was not associated with DL $(\mathrm{aOR}=1.02,95 \% \mathrm{CI}=0.64-1.64)$.

Available cross-sectional studies have not reached a consensus concerning the relationship between sleep duration and DL. For example, Lin et al. $(2017)^{10}$ and Choi et al. (2008) ${ }^{11}$ utilizing self-reported questionnaires revealed a U-shaped association between sleep duration and low HDL-C among middle-aged and elderly population in Taiwan and sleep duration and low HDL-C and high TG levels among Koreans over the age of 60, respectively. On the other hand, Bjorvatn et al. $(2007)^{12}$ demonstrated that in individuals from the Hordaland County, Norway, TC, and TG levels were higher only among short sleepers. Similarly, Smiley et al. (2019) concluded that short sleep duration was related to high TG and low HDL-C levels among US citizens that participated in the 2013-14 National Health and Nutrition Examination Survey (NHANES). In contrast, Shin et al. (2016) ${ }^{13}$ exhibited that long, but not short sleep duration was related to low HDL-C levels among a Korean adult population from the Korean National Health and Nutrition Examination Survey. Van Den Berg et al. (2008) ${ }^{48}$ also found that long sleep duration was positively associated with DL investigating a sample of 768 elderly adults from the Netherlands.

Our study did not elicit gender-specific associations between short or long sleep duration and DL. In keeping with our results, Song et al. $(2020)^{18}$ could also not find significant interactions between sleep duration and sex with respect to abnormal serum lipid levels. In contrast, data from the China Health and Nutrition Survey (2009) showed that both short and long sleep duration were associated with higher risks of abnormal serum lipid profiles in women, but not in $\mathrm{men}^{17}$. Similarly, Kaneita et al. (2008) ${ }^{43}$ found that both short and long sleep duration were positively associated with high TG and low HDL-C in Japanese females, but not males. Nakanishi et al. $(1999)^{44}$ could also not find a significant association between sleep duration and serum lipid and lipoprotein levels among Japanese men. Finally, Williams et al. $(2007)^{45}$ exhibited that short sleep duration was related to low HDL-C levels in adult American women with type 2 diabetes. Several investigators have tried to explain the gender-specific effects forging the relationship between sleep quantity and DL by implicating bias in self-reporting of sleep ${ }^{46}$ and differences born either by social and household statutes ${ }^{47}$ or by sex hormones variation ${ }^{48}$.

With regards to sleep quality, DL remained significantly associated only with insomnia $(\mathrm{aOR}=1.43, p=0.050)$ while its relation with poor sleep quality $(\mathrm{aOR}=1.31, p=0.094)$ and the risk for OSA $(\mathrm{aOR}=1.32, p=0.097$ ) were found of marginal statistical significance. However, in stratified analysis according to gender, DL was significantly associated with insomnia $(\mathrm{aOR}=1.75,95 \% \mathrm{CI}=1.05$ 2.94, $p=0.030)$, poor sleep quality $(\mathrm{aOR}=2.02,95 \% \mathrm{CI}=1.23$ $3.33, p=0.006)$ and the risk for OSA $(\mathrm{aOR}=1.62,95 \% \mathrm{CI}=0.97-2.72$, $p=0.067)$ in females but not males. Concerning insomnia subtypes DL was significantly associated with difficulties maintaining sleep $(\mathrm{aOR}=2.99, p<0.001)$ and early morning awakenings $(\mathrm{aOR}=1.38$, $p=0.050)$, but not difficulties initiating sleep ( $\mathrm{aOR}=1.18, p=0.328$ ). Again, DL was associated with difficulties maintaining sleep (aOR=6.84, 95\% CI=3.86-12.11, $p<0.001$ ) and early morning awakenings ( $\mathrm{aOR}=1.70,95 \% \mathrm{CI}=1.08-2.68, p=0.022$ ) solely among females, whereas only females exhibited a marginally significant association between DL and difficulties initiating sleep $(\mathrm{aOR}=1.54$, 95\% CI=0.94-2.52, $p=0.089)$. Few studies have investigated the relationship between sleep quality and insomnia. Vozoris (2016) ${ }^{16}$ having used data from the 2005-2006 and 2007-2008 National Health and Nutrition Examination Surveys (NHANES) concluded that insomnia symptoms are not associated with DL. Similarly, Zhan et al. (2014) utilizing data from more than 10.000 Chinese individuals, apart from $25 \%$ increased odds of elevated TC level only among women experiencing insomnia, did not find significant associations between insomnia and LDL-C, HDL-C, or TG levels among both sexes. In contrast, Rouleau et al. (2017) ${ }^{49}$ exhibited that upon completion of an exercise-based cardiac rehabilitation program, greater improvement in insomnia symptom severity was associated with greater improvements in TC levels. Finally, Chien et al. (2010) ${ }^{50}$ revealed that Taiwanese insomniacs had, surprisingly, lower TC levels compared to non-insomniacs.

Several mechanisms have been implicated in the pathogenetic relationship between inadequate sleep and DL. The study of Corgosinho et al. (2013) $)^{51}$ highlighted the burden of short sleep duration on human metabolism, thus inducing increased dietary fat consumption, DL and obesity, which is many times difficult to confront especially among adolescents. For example, sleep restriction strongly influences metabolic hormones that regulate energy balance, as it has been shown that short sleep duration lowers the blood concentration of leptin that suppresses appetite, and increases the blood concentration of ghrelin, which promotes appetite and sequentially daily dietary intake of cholesterol, trans-fats, and saturated fats ${ }^{52}$. Apart from that, inadequate sleep has been associated with excessive daytime fatigue and consequent reduced engagement in physical activities. Low levels of physical activity have been shown to increase LDL and lower HDL levels ${ }^{53}$. Moreover, it has been presented that short sleep duration by increasing acute stress significantly raises TC and LDL-C levels ${ }^{54}$. In agreement, the evidence of Andersen et al. (2009) ${ }^{55}$ on animal models 
suggest that the exposure to chronic stressful stimuli resulted in impairment of all parameters of the lipid profile. Finally, it has been proposed that sleep restriction could modify genetic risk factors for adverse blood lipid profiles ${ }^{56}$.

Our analysis manifests several strengths, as it is based on data from a large representative sample of the population in northeastern Greece that provided excellent response rates to sleep quality and quantity and DL measurements (71\% compared, for example, to $63 \%$ in the Hordaland Health Study $)^{12}$. Moreover, our sampling scheme ensured that the sample was randomly selected and representative of the general population of this area. Limitations of this study lie in the recall bias of self-reported sleep duration as reported sleep duration may be overestimated ${ }^{57}$. Nevertheless, self-report assessments of sleep have been shown to be valid measures compared to quantitative ones based on actigraphy ${ }^{58}$. Apart from that, data were not obtained separately on hypercholesterolemia or hypertrygliceridemia or in patients who were on treatment for dyslipidemia, and therefore the relationship between sleep disturbances with these two dyslipidemic states or patients who were on lipid-lowering agents could not be evaluated. Another limitation involves the properties of the cross-sectional study as causal relationships between sleep pathology and DL could not be determined.

Heterogeneity in sleep duration definition combined with the limited number of relevant studies performed ${ }^{59,60}$ constitute the evidence based statistical interpretation a tedious $\operatorname{task}^{61}$. Thus, further prospective studies are required and a longitudinal study on this topic by our research team is scheduled.

\section{CONCLUSION}

To the best of our knowledge, this is the first conducted cross-sectional study in Greece that elucidates an association between short sleep duration, insomnia and DL. Considering its strengths and limitations it could be proposed that early pharmacological, cognitive, and behavioral interventions that improve sleep quality and quantity might serve as preventative measures for DL.

\section{ACKNOWLEDGMENTS}

The contributions of all of the participants, patient advisers, and interviewers are gratefully acknowledged.

\section{SOURCES OF FUNDING}

This research did not receive any specific grant from funding agencies in the public, commercial, or not-for-profit sectors.

\section{CONFLICTS OF INTEREST}

The authors report no conflicts of interest

\section{REFERENCES}

1. GBD 2015 Mortality and Causes of Death Collaborators. Global, regional, and national life expectancy, all-cause mortality, and cause-specific mortality for 249 causes of death, 1980-2015: a systematic analysis for the Global Burden of Disease Study ２015. Lancet. 2016 Oct;388(10053):1459-544. DOI: https://doi.org/10.1016/S0140-6736(16)31012-1

2. Lennon RP, Claussen KA, Kuersteiner KA. State of the heart: an overview of the disease burden of cardiovascular disease from an epidemiologic perspective. Prim Care. 2018 Mar;45(1):1-15. DOI: https://doi.org/10.1016/j.pop.2017.11.001
3. Third Report of the National Cholesterol Education Program (NCEP). Expert panel on detection, evaluation, and treatment of high blood cholesterol in adults (Adult Treatment Panel III) final report. Circulation. 2002 Dec;106(25):3143-421.

4. O'Malley PG, Arnold MJ, Kelley C, Spacek L, Buelt A, Natarajan S, et al. Management of dyslipidemia for cardiovascular disease risk reduction: synopsis of the 2020 Updated U.S. Department of Veterans Affairs and U.S. Department of Defense Clinical Practice Guideline. Ann Intern Med. 2020 Nov;173(10):822-9. DOI: https://doi.org/10.7326/M20-4648

5. Choudhury SR, Ueshima H, Kita Y, Kobayashi KM, Okayama A, Yamakawa $\mathrm{M}$, et al. Alcohol intake and serum lipids in a Japanese population. Int J Epidemiol. 1994 Oct;23(5):940-7. DOI: https://doi. org/10.1093/ije/23.5.940

6. Hata Y, Nakajima K. Life-style and serum lipids and lipoproteins. J Atheroscler Thromb. 2000;7(4):177-97. DOI: https://doi.org/10.5551/ jat1994.7.177

7. Shivashankar R, Kondal D, Ali MK, Gupta R, Pradeepa R, Mohan V, et al. Associations of sleep duration and disturbances with hypertension in Metropolitan Cities of Delhi, Chennai, and Karachi in South Asia: cross-sectional analysis of the CARRS study. Sleep. 2017;40(9):zsx119. DOI: https://doi.org/10.1093/sleep/zsx119

8. Gangwisch JE, Heymsfield SB, Boden-Albala B, Buijs RM, Kreier F, Pickering TG, et al. Sleep duration as a risk factor for diabetes incidence in a large US sample. Sleep. 2007 Dec;30(12):1667-73. DOI: https:// doi.org/10.1093/sleep/30.12.1667

9. Cappuccio FP, Cooper D, D’Elia L, Strazzullo P, Miller MA. Sleep duration predicts cardiovascular outcomes: a systematic review and meta-analysis of prospective studies. Eur Heart J. 2011 Jun;32(12):148492. DOI: https://doi.org/10.1093/eurhearti/ehr007

10. Lin P, Chang KT, Lin YA, Tzeng IS, Chuang HH, Chen JY. Association between self-reported sleep duration and serum lipid profile in a middle-aged and elderly population in Taiwan: a community-based, cross-sectional study. BMJ Open. 2017;7(10):e015964. DOI: https:// doi.org/10.1136/bmjopen-2017-015964

11. Choi KM, Lee JS, Park HS, Baik SH, Choi DS, Kim SM. Relationship between sleep duration and the metabolic syndrome: Korean National Health and Nutrition Survey 2001. Int J Obes (Lond). 2008 May;32(7):1091-7. DOI: https://doi.org/10.1038/ijo.2008.62

12. Bjorvatn B, Sagen IM, Øyane N, Waage S, Fetveit A, Pallesen S, et al. The association between sleep duration, body mass index and metabolic measures in the Hordaland Health Study. J Sleep Res. 2007 Mar;16(1):6676. DOI: https://doi.org/10.1111/j.1365- 2869.2007.00569.x

13. Shin HY, Kang G, Kim SW, Kim JM, Yoon JS, Shin IS. Associations between sleep duration and abnormal serum lipid levels: data from the Korean National Health and Nutrition Examination Survey (KNHANES). Sleep Med. 2016 Aug;24:119-23. DOI: https://doi. org/10.1016/j.sleep.2016.05.012

14. Chien KL, Chen PC, Hsu HC, Su TC, Sung FC, Chen MF, et al. Habitual sleep duration and insomnia and the risk of cardiovascular events and all-cause death: report from a community-based cohort. Sleep. 2010 Feb;33(2):177-84. DOI: https://doi.org/10.1093/sleep/33.2.177

15. Zhan Y, Zhang F, Lu L, Wang J, Sun Y, Ding R, et al. Prevalence of dyslipidemia and its association with insomnia in a community based population in China. BMC Public Health. 2014 Oct;14(1):1050. DOI https://doi.org/10.1186/1471-2458-14-1050

16. Vozoris NT. Insomnia symptoms are not associated with dyslipidemia: a population-based study. Sleep. 2016 Mar;39(3):551-8. DOI: https:// doi.org/10.5665/sleep.5524

17. Zhan Y, Chen R, Yu J. Sleep duration and abnormal serum lipids: the China Health and Nutrition Survey. Sleep Med. 2014;15(7):833-9. DOI: https://doi.org/10.1016/j.sleep.2014.02.006

18. Song Q, Liu X, Zhou W, Wu S, Wang X. Night sleep duration and risk of each lipid profile abnormality in a Chinese population: a prospective cohort study. Lipids Health Dis. 2020 Aug;19(1):185. DOI: https://doi. org/10.1186/s12944-020-01363-y

19. Potter GDM, Cade JE, Hardie LJ. Longer sleep is associated with lower BMI and favorable metabolic profiles in UK adults: findings from the National Diet and Nutrition Survey. PLoS One. 2017;12(7):e0182195. DOI: https://doi.org/10.1371/journal.pone.0182195

20. Serdari A, Manolis A, Tsiptsios D, Vorvolakos T, Terzoudi A, Nena $E$, et al. Insight into the relationship between sleep characteristics and anxiety: a cross-sectional study in indigenous and minority populations in northeastern Greece. Psychiatry Res. 2020 Oct;292:113361. DOI: https://doi.org/10.1016/j.psychres.2020.113361

21. Vorvolakos T, Leontidou E, Tsiptsios D, Mueller C, Serdari A, Terzoud A, et al. The association between sleep pathology and depression: a cross-sectional study among adults in Greece. Psychiatry R e s 2020;294:113502. DOI: https://doi.org/10.1016/j.psychres.2020.113502 
22. Matziridis A, Tsiptsios D, Manolis A, Ouranidis A, Triantafyllis AS, Tsamakis K, et al. Sleep insufficiency and incident diabetes mellitus among indigenous and minority populations in Greece. Sleep Sci. 2020 Dec;294:113502.

23. Tsiptsios D, Matziridis A, Ouranidis A, Triantafyllis AS, Terzoudi A, Tsamakis $\mathrm{K}$, et al. The age and gender effect on the association of sleep insufficiency with hypertension among adults in Greece. Future Cardiol. 2021 Mar 01; [Epub ahead of print]. DOI: https://doi. org/10.2217/fca-2020-0198

24. Lakerveld J, Mackenbach JD, Horvath E, Rutters F, Compernolle S, Bárdos $\mathrm{H}$, et al. The relation between sleep duration and sedentary behaviours in European adults. Obes Rev. 2016 Feb;17(Suppl 1):62-7. DOI: https://doi.org/10.1111/obr.12381

25. Stranges S, Dorn JM, Shipley MJ, Kandala NB, Trevisan M, Miller MA, et al. Correlates of short and long sleep duration: a cross-cultural comparison between the United Kingdom and the United States: the Whitehall II Study and the Western New York Health Study. Am J Epidemiol. 2008 Dec;168(12):1353-64. DOI: https://doi.org/10.1093/aje/kwn337

26. Tsara V, Serasli E, Amfilochiou A, Constantinidis T, Christaki P. Greek Version of the Epworth sleepiness scale. Sleep Breathing. 2004 Apr;8(2):91-5. DOI: https://doi.org/10.1007/s11325-004-0091-6

27. Soldatos CR, Dikeos DG, Paparrigopoulos TJ. Athens insomnia scale: validation of an instrument based on ICD-10 criteria. J Psychosomatic Res. 2000 Jun;48(6):555-60. DOI: https://doi.org/10.1016/s00223999(00)00095-7

28. Kotronoulas GC, Papadopoulou CN, Papapetrou A, Patiraki E. Psychometric evaluation and feasibility of the Greek Pittsburgh sleep quality index (GR-PSQI) in patients with cancer receiving chemotherapy. Support Care Cancer. 2011 Oct;19(11):1831-40. DOI: https://doi.org/10.1007/s00520-010-1025-4

29. Bouloukaki I, Komninos ID, Mermigkis C, Micheli K, Komninou M, Moniaki V, et al. Translation and validation of Berlin questionnaire in primary health care in Greece. BMC Pulmonary Medicine. 2013 Jan;13(1):6. DOI: https://doi.org/10.1186/1471-2466-13-6

30. Vinueza R, Boissonnet CP, Acevedo M, Uriza F, Benitez FJ, Silva H, et al. Dyslipidemia in seven Latin American cities: CARMELA study. Prev Med. 2010 Mar;50(3):106-11. DOI: https://doi.org/10.1016/j. ypmed.2009.12.011

31. Milias GA, Panagiotakos DB, Pitsavos C, Xenaki D, Panagopoulos G, Stefanadis C. Prevalence of self-reported hypercholesterolaemia and its relation to dietary habits, in Greek adults; a national nutrition \& health survey. Lipids Health Dis. 2006 Mar;5(1):5. DOI: https://doi. org/10.1186/1476-511X-5-5

32. Merekoulias G, Sahount E, Chalimou I, Loukopoulou P, Lianas D. Sleep disorders in elderly rural population of Western Greece. Achaiki Iatriki. 2014 Jul;33(2):95-103.

33. Hirode G, Vittinghoff E, Bharmal NH, Kandula NR, Kanava AM The association of religious affiliation with cholesterol levels among South Asians: the Mediators of Atherosclerosis in South Asians Living in America study. BMC Cardiovasc Disord. 2019 Mar;19(1):75. DOI: https://doi.org/10.1186/s12872-019-1045-z

34. Al Sifri SN, Almahmeed W, Azar S, Okkeh O, Bramlage P, Jünger C, et al. Results of the Dyslipidemia International Study (DYSIS)-Middle East: clinical perspective on the prevalence and characteristics of lipid abnormalities in the setting of chronic statin treatment. PLoS One. 2014 Jan;9(1):e84350. DOI: https://doi.org/10.1371/journal.pone.0084350

35. Nam GE, Cho KH, Park YG, Han KD, Choi YS, Kim SM, et al. Socioeconomic status and dyslipidemia in Korean adults: the 2008-2010 Korea National Health and Nutrition Examination Survey. Prev Med. 2013 Oct;57(4):304-9. DOI: https://doi.org/10.1016/j.ypmed.2013.06.008

36. Tousoulis D, Oikonomou E, Vogiatzi G, Vardas P. Cardiovascular disease and socioeconomic status. Eur Heart J. 2020 Sep;41(34):3213-4. DOI: https://doi.org/10.1093/eurheartj/ehaa405

37. Nikparvar M, Yousefi $H$, Kheirandish M. Dyslipidemia and its associated factors in women of the Bandare-Kong cohort study. Res Square. 2020 Aug 12; [Epub preprint]. DOI: https://doi.org/10.21203/ rs.3.rs- $56630 / \mathrm{v} 1$

38. Ni WQ, Liu XL, Zhuo ZP, Yuan XL, Song JP, Chi HS, et al. Serum lipids and associated factors of dyslipidemia in the adult population in Shenzhen. Lipids Health Dis. 2015 Jul;14:71. DOI: https://doi. org/10.1186/s12944-015-0073-7

39. Banitalebi M, Rouzbahani R, Motlagh RKN. Nutrition and physical activity in Iranian dyslipidemic patients. Acta Med Iran. 2019;57(10):592-7.

40. Rekleiti M, Hatzinikolaou V, Saridi M, Kyloudis P, Wozniak G, Skliros E, et al. P-525 - Investigating stress and depression within a rural population group in Greece: a pilot study. Eur Psychiatry. 2012 Dec;27:1.
41. Smiley A, King D, Harezlak J, Dinh P, Bidulescu A. The association between sleep duration and lipid profiles: the NHANES 2013-2014. J Diabetes Metab Disord. 2019 Jun;18(2):315-22. DOI: https://doi. org/10.1007/s40200-019-00415-0

42. Van Den Berg JF, Miedema HM, Tulen JH, Neven AK, Hofman A, Witteman JC, et al. Long sleep duration is associated with serum cholestero in the elderly: the Rotterdam study. Psychosom Med. 2008 Nov;70(9):100511. DOI: https://doi.org/10.1097/PSY.0b013e318186e656

43. Kaneita Y, Uchiyama M, Yoshiike N, Ohida T. Associations of usual sleep duration with serum lipid and lipoprotein levels. Sleep. 2008 May;31(5):645-52. DOI: https://doi.org/10.1093/sleep/31.5.645

44. Nakanishi N, Nakamura K, Ichikawa S, Suzuki K, Tatara K. Relationship between lifestyle and serum lipid and lipoprotein levels in middle-aged Japanese men. Eur J Epidemiol. 1999 Apr;15(4):341-8. DOI: https://doi. org/10.1023/a:1007527111946

45. Williams CJ, Hu FB, Patel SR, Mantzoros CS. Sleep duration and snoring in relation to biomarkers of cardiovascular disease risk among women with type 2 diabetes. Diabetes Care. 2007 May;30(5):1233-40. DOI: https://doi.org/10.2337/dc06-2107

46. Baldwin CM, Kapur VK, Holberg CJ, Rosen C, Nieto FJ; Sleep Health Study Group. Associations between gender and measures of daytime somnolence in the Sleep Heart Health Study. Sleep. 2004;27(2):305-11. DOI: https://doi.org/10.1093/sleep/27.2.305

47. Park YM, Matsumoto K, Shinkoda H, Nagashima H, Kang MJ, Seo YJ Age and gender difference in habitual sleep-wake rhythm. Psychiatry Clin Neurosci. 2008 Sep;55(3):201- 2. DOI: https://doi.org/10.1046/ j.1440-1819.2001.00825.x

48. Dzaja A, Arber S, Hislop J, Kerkhofs M, Kopp C, Pollmächer T, et al. Women's sleep in health and disease. J Psychiatr Res. 2005 Jan;39(1):5576. DOI: https://doi.org/10.1016/j.jpsychires.2004.05.008

49. Rouleau CRTK, Aggarwal S, Arena R, Campbell TS. The association between insomnia symptoms and cardiovascular risk factors in patients who complete outpatient cardiac rehabilitation. Sleep Med. 2017 Apr;32:201-7. DOI: https://doi.org/10.1016/j.sleep.2017.01.005

50. Chien KL, Chen PC, Hsu HC, Su TC, Sung FC, Chen MF, et al. Habitual sleep duration and insomnia and the risk of cardiovascular events and all-cause death: report from a community-based cohort. Sleep. 2010 Feb;33(2):177-84. DOI: https://doi.org/10.1093/sleep/33.2.177

51. Corgosinho FC, Dâmaso AR, Ganen AP, Campos RMS, Silva PL, Sanchez PL, et al. Short sleep time increases lipid intake in obese adolescents. Sleep Sci. 2013;6(1):26-31.

52. Taheri S, Lin L, Austin D, Young T, Mignot E. Short sleep duration is associated with reduced leptin, elevated ghrelin, and increased body mass index. PLoS Med. 2004;1(3):e62. DOI: https://doi.org/10.1371/journal.pmed.0010062

53. Haskell WL. The influence of exercise on the concentrations of triglyceride and cholesterol in human plasma. Exerc Sport Sci Rev. 1984;12:205-44.

54. Bachen EA, Muldoon MF, Matthews KA, Manuck SB. Effects of hemoconcentration and sympathetic activation on serum lipid responses to brief mental stress. Psychosom Med. 2002 Jul/Aug;64(4):587-94. DOI: https://doi.org/10.1097/01.psy.0000021943.35402.8a

55. Andersen ML PJ, Bignotto M, Tufik S. Differential effects of sleep loss and chronic stressors on lipid metabolism. Sleep Sci. 2009;2(3):135-40.

56. Noordam R, Bos MM, Wang H, Winkler TW, Bentley AR, Kilpeläinen TO, et al. Multi-ancestry sleep-by-SNP interaction analysis in 126,926 individuals reveals lipid loci stratified by sleep duration. Nat Commun. 2019 Nov;10(1):5121. DOI: https://doi.org/10.1038/s41467-019-12958-0

57. Lauderdale DS, Knutson KL, Yan LL, Rathouz PJ, Hulley SB, Sidney $\mathrm{S}$, et al. Objectively measured sleep characteristics among early-middleaged adults: the CARDIA study. Am J Epidemiol. 2006 Jul;164(1):5-16. DOI: https://doi.org/10.1093/aje/kwj199

58. Lockley SW, Skene DJ, Arendt J. Comparison between subjective and actigraphic measurement of sleep and sleep rhythms. J Sleep Res. 2002 Jan;8(3):175-83. DOI: https://doi.org/10.1046/j.13652869.1999.00155.x

59. Gangwisch JE, Malaspina D, Babiss LA, Opler MG, Posner K, Shen S, et al. Short sleep duration as a risk factor for hypercholesterolemia: analyses of the National Longitudinal Study of Adolescent Health. Sleep. 2010 Jul;33(7):956-61. DOI: https://doi.org/10.1093/sleep/33.7.956

60. Petrov ME, Kim Y, Lauderdale D, Lewis CE, Reis JP, Carnethon MR, et al. Longitudinal associations between objective sleep and lipids: the CARDIA study. Sleep. 2013 nov;36(11):1587-95. DOI: https://doi. org $/ 10.5665 /$ sleep.3104

61. Kruisbrink M, Robertson W, Ji C, Miller MA, Geleijnse JM, Cappuccio FP. Association of sleep duration and quality with blood lipids: a systematic review and meta- analysis of prospective studies. BMJ Open. 2017;7(12):e018585. DOI: https://doi.org/10.1136/ bmjopen-2017-018585 